\title{
Star Formation in Interacting Galaxies
}

\author{
Curtis Struck \\ Dept. of Physics and Astronomy, Iowa State University \\ Ames, IA 50011 USA \\ email: curt@iastate.edu
}

\begin{abstract}
This brief review emphasizes the wide range of environments where interaction induced star formation occurs. In these environments we can study the numerous elaborations of a few basic physical processes, including: gravitational instability, accretion and large-scale shocks.
\end{abstract}

Keywords. galaxies: interactions, galaxies: evolution, stars: formation

\section{Introduction and Background}

This has become a huge topic in the last few decades, with many specific areas of active research. Induced star formation in major mergers produces the most luminous galaxies in the universe, and its cumulative contribution to the net star formation (SF) of typical galaxies is substantial. The amount and type of star formation induced in collisions and mergers depends on environment and cosmological time, with important corollary phenomena like downsizing and the peak in the cosmological SF rate at redshifts of 2-3. The disequilibrium of disks, and the production of tidal structures like bridges, tails and shells, provide unique environments for star formation, e.g, in tidal dwarf galaxies, and proto-globular clusters. (The former are distinguished from the latter by greater masses, e.g., $\geqslant 10^{7} M_{\odot}$.) Interactions also compress and funnel gas into galaxy cores driving a range of activities therein.

This large range of topics cannot be covered in this brief review. Dynamical forces acting on galaxy scales (typically $3-30 \mathrm{kpc}$ ) are primarily responsible for the induced star formation, so I will focus the discussion on these scales. The associated phenomena on the smaller scales of molecular clouds, and the larger scales of cosmological structure formation are considered in other reviews at this meeting. Similarly, I will generally not discuss computational methods, but concentrate on what has been learned from the interplay between observations, models and analytic theory. In some systems with unique SF structures these comparisons can strongly constrain model treatments. The topics that I do want briefly visit include: some special star-forming environments created in collisions between two comparable galaxies, which can be considered playgrounds for the study of induced star formation (ISF), explorations of dynamically complex environments in groups, attempts to automate parts of the dynamical modeling, and the state of analytic theory.

Before probing these specific topics, I would like to review a little background on the big questions in this field. First is the question of how much of the net cosmic star formation can be blamed on interactions and mergers? The last decade has seen a great deal of progress towards answering this from both deep observational surveys, and cosmological structure buildup simulations. Examples of the former include the work of Jogee, et al. (2009), who examined data from GEMS, Combo-17 and Spitzer deep fields, with sensitivity out to $z \simeq 0.8$. Their overall conclusion was that mergers major and minor 
can account for $\leqslant 30 \%$ of the star formation in the cosmic time range of 3-7 Gyr ago. As a second example, Shi, et al. (2009) in a study of LIRGS in the GOODS survey conclude that the fraction of mergers among these strongly star-forming objects is about $50 \%$ up to a redshift of $z \simeq 1.2$. Studies of both the cosmic SFR and the merger rate predict increases out to redshifts of 2-3, so we can expect the interaction induced star formation is even higher at those redshifts. There is little question that interaction/merger ISF is a very important contributor. However, it is also clear that we have some ways to go in order to have accurate, quantitative estimates of the amount in different cosmological epochs.

Other big questions include where, when and how the interaction-induced SF occurs? A significant part of these questions was answered a couple of decades ago with IRAS observations. That data led to the discovery of ULIRGS, to the recognition that most SF occurs in the final phases of wet merging, and that it is buried deep within the nucleus of the remnant. This was summarized in the conference proceedings of Sulentic, Keel, \& Telesco (1990), and later explained in part by numerical simulations (e.g., Barnes \& Hernquist 1996, Mihos \& Hernquist 1996). The high redshift version of that story is being elaborated steadily at the present time.

What about ISF before the merger? A number of surveys have examined this question in the last decade, usually comparing extensive samples of paired versus unpaired galaxies (see the reviews of Struck 2006, and in Smith, et al. 2010a), or samples of obviously interacting galaxies to comparable isolated objects. Weaknesses of the former technique include the fact a fraction of the paired galaxies may not be interacting (even with very similar recession velocities), and minor companions of comparison objects may not always be visible. Both effects are probably small in recent studies. In the case of strongly interacting samples, the difficulties are often associated with obtaining data on a large sample of the rather rare objects. Yet studies with the two techniques seem to be coming to similar conclusions. The result is that SF is enhanced in pre-merger interactions, but only by a factor of a few or less, and this enhancement is often dominated by nuclear starbursts in one of the pair, at a time near closest approach. However, there are a number of less typical cases worthy of study.

\section{Star-forming Environments in Galaxy Collisions}

Among the specific reasons for considering these examples is firstly, to understand differences and similarities between star-forming regions in the Local Group and more extreme environments. Secondly, given that we still must use phenomenological prescriptions for SF in models on these and larger scales, it is worth briefly considering how some these apply over the range of observed conditions. Let's begin with some gaseous shocks with an extent and intensity definitely not seen in our galaxy.

I will pass by one of the most famous, the "Overlap Region" in the Antennae system, if only because of the angle at which we view it is not ideal, making observational interpretations difficult. Several posters were presented at this meeting on Antennae models by Chapon, Karl, Kruijssen, and Renaud (see summaries in this volume). Each of these has a different and interesting angle on the system.

Consider instead the shocks in the ocular ring in the disk of IC 2163, which both observations and models (Struck 2005) suggest has been orbiting around its companion, NGC 2207 for some time. Most of the emission in various bands comes from strong $\mathrm{SF}$ in the ocular wave, rather than directly from the large-scale shocks. However, the sharp dust lanes in HST observations, and strong dust emission in the Spitzer Space Telescope observations, are indicative of such shocks. Because of its proximity this galaxy 
is probably the best system for detailed study of such waves. It apparently has another very large shock to offer. An ongoing study of a unique band of radio continuum emission at the interface between the two disks suggests it may be the result of gentle scraping, as the disks almost or just barely touch (see Fig. 1 Kaufman, et al. 2010, ). In contrast to the Antennae overlap, this soft encounter evidently induces virtually no SF.

One of the most beautiful shocks in an interacting system is found in Arp 118, investigated by Appleton, et al. (2003), see the right panel in Fig. 1. This shock is revealed in optical imagery as a long, multi-kiloparsec dust lane. By a fluke of nature we have amazingly direct evidence that this is a shock. Absorption against a background radio jet allows us to see the $185 \mathrm{~km} / \mathrm{s}$ shock velocity jump in HI absorption. There is little star formation over most of the length of the shock, but Appleton et al. presented a plausible case that the shock compression does induce star formation, with a time delay of about $25 \mathrm{Myr}$, and which is visible at a considerable distance downstream. The shock itself is a type that is commonly seen to develop at the leading edge of a tidal arm in models (e.g., in the semi-analytical models of Gerber \& Lamb 1994). In other systems, star formation does occur in such arms, especially at latter stages when the material is flung out to greater distances, and arm material travels with the feature rather than moving through it.

An extreme example of strong, large-scale shocks in interacting systems is found in the so-called Taffy system (UGC 12914/15, see Gao, Zhu \& Seaquist 2003), and its several known sister systems. Here a nearly direct collision between galaxy disks leaves gas and magnetic fields strewn between the two. In the Taffy there is little SF in the debris field, despite the fact that much of the gas there must have been strongly shocked. There is $\mathrm{SF}$ in and near the disks, which may include infalling gas.

The assumption that SF is directly induced by shocks has been used as the basis of phenomenological SF laws in simulations. J. Barnes has recently advocated this procedure (see e.g., Chien \& Barnes 2009), and has provided numerical examples to show that such a prescription does a better job than gas density-dependent forms in matching the SF
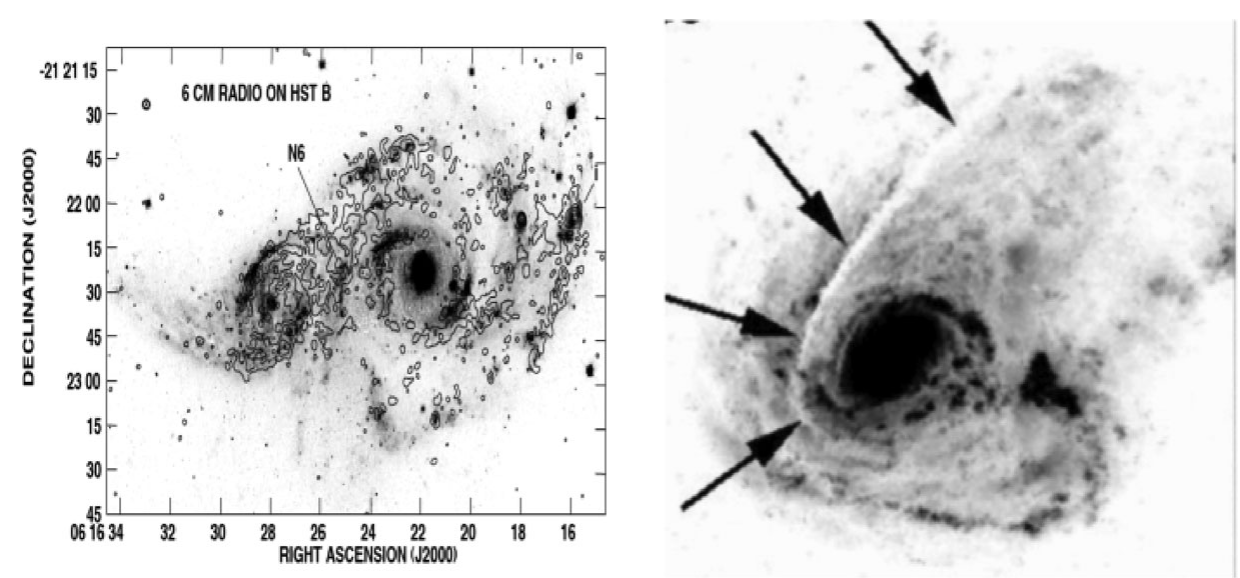

Figure 1. Examples of large-scale shocks with little apparent SF. The NGC 2207/IC 2163 system is shown on the left (from Kaufman, et al. 2010). An optical image (HST B-band) is shown in grayscale, and radio continuum emission is shown as contours. Note the continuum emission between the galaxies. An optical (HST) image of Arp 118 is shown on the right (from Appleton, et al. 2003). The large scale shock is highlighted by a long thin dust lane. The induced star formation lies off the shock. Note: according to the NASA Extragalactic Database, the scale of the system is $560 \mathrm{pc} /$ arcsec. 
in a couple of well-known merger remnants. In light of the above examples I would caution that there may be subtleties in other cases. Some of these examples suggest that there may a threshold compression before shocks can effectively trigger SF, and the Arp 118 example suggests that the time delays may play a role in some cases. Recent high resolution numerical studies shed further light on these questions (see Kim, et al. 2009, Saitoh, et al. 2009, Teyssier, et al. 2010), and some of these works were updated at this meeting.

Another set of processes for triggering SF are gravitational or magneto-gravitional instabilities (see e.g., Elmegreen 1989, Efremov 2010). A characteristic and oft-cited symptom of this disease is regularly spaced clumps of young stars, or molecular clouds, or HI superclouds the so-called beads-on-a-string. The beads are relatively rare in isolated galaxies, but interactions can trigger them on large scales. It is my impression that they are not quite so rare in colliding galaxies. The Spitzer/IRAC images of the NGC 2207/IC 2163 system show them in a number of arm sections. HST reveals optical subclumps in some of them (Elmegreen, et al. 2006 and references therein).

They are apparently quite common in M51-type flyby systems, in the spirals or extended tails. This includes M51 itself, but other morphologically similar examples are: Arp 72, Arp 82, and Arp 86. The beaded structure in bridges and tails is often more prominent and extensive in GALEX UV observations (see Fig. 2 from Smith, et al. 2010b).

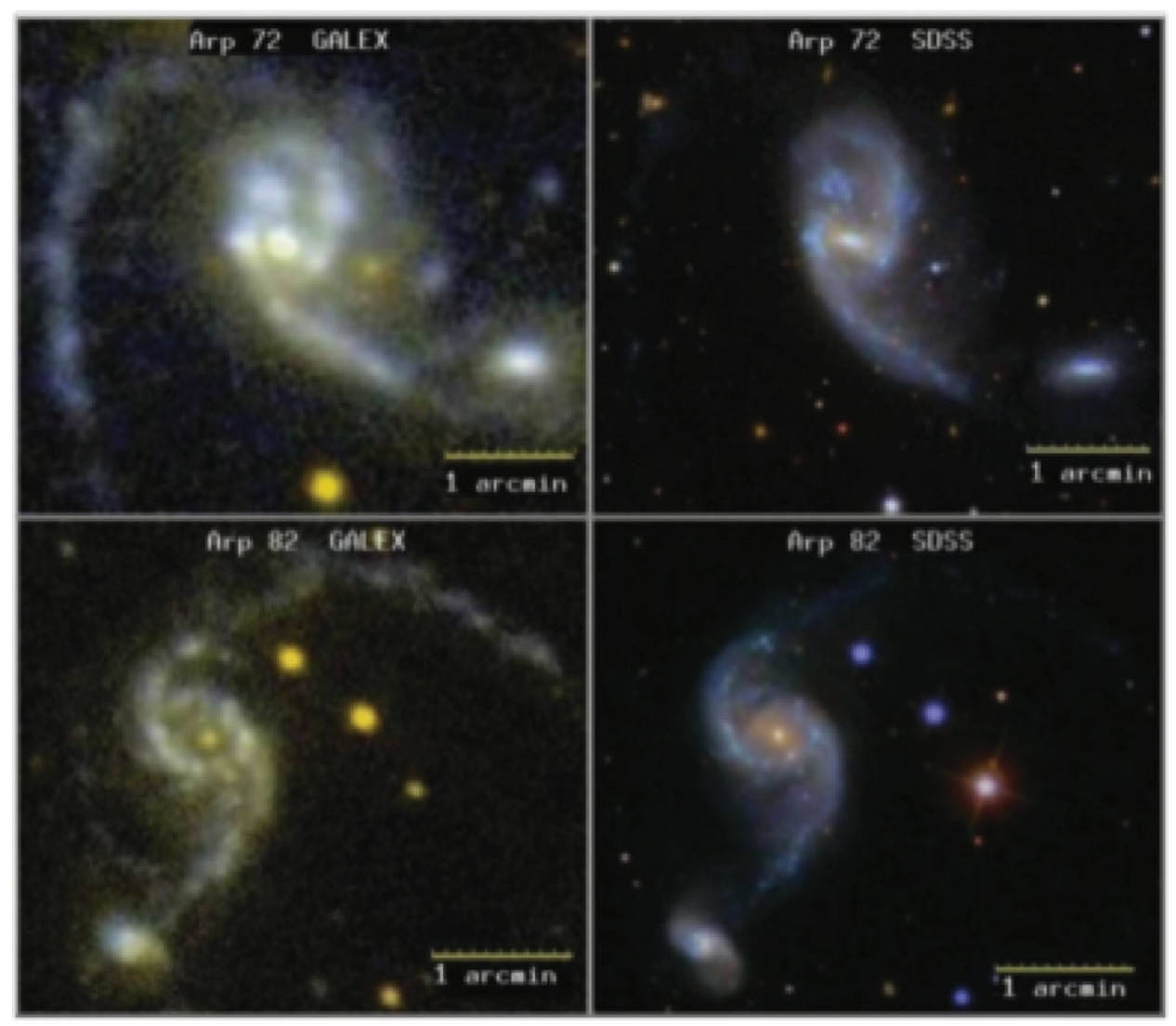

Figure 2. Sample beads-on-a-string in the tidal tails of Arp 72 and Arp 82 (from Smith, et al. 2010b). The beads are much more prominent in the GALEX UV images on the left, than in the SDSS images on the right. 
Some colliding ring galaxies, like the Cartwheel are also beaded. What these types have in common are strong waves, or as in the case of material tails, material that remains in proximity long enough to pull together under self-gravity.

The beads often have substructure. Thus, they do not result from an instability with a single dominant wavelength. The concepts of "competitive accretion" within the waves, or a tidal wave version of "collect and collapse" may operate on a timescale set by each unique environment. Numerical models now have the resolution to test such notions.

A third process, besides shock compression and gravitational instability, which likely plays an important role in interaction induced star formation is accretion or pile-up. There are many kinds of accretion on galaxy scales. Cosmological accretion has excited a great deal of interest lately as a competitor to ISF in mergers, but is beyond the scope of this review. Gas concentration in wet mergers can also be viewed as accretion, but the topic has been frequently reviewed before. I will survey some examples in ongoing interactions.

The most well known example of recent years is the Duc-Bournaud theory of tidal dwarf galaxy (TDG) formation (see Duc, Bournaud, \& Masset 2004, Bournaud, \& Duc 2006). Their models demonstrated that TDGs form in a pile-up at the end of tidal tail, where material is unable to push any farther out of the joint dark halo of the merging galaxies. Its hard to get much material out that far, so only dwarfs are made. However, as shown by the models it can accumulate for $\simeq 10^{8}$ yr., pull together gravitationally, and form stars. According to Lisenfeld, et al. (2009) the observed correlation between molecular gas density and SFR obeys the Schmidt Law in these objects. But only a handful of objects to date have observations sufficient to test this.

A similar pileup occurs when bridge material swings around a companion as in Arp 285 (see Fig. 3 and Smith, et al. 2008). Like the beads phenomenon, SF occurs in a line of clumps in this region, but simulations suggest that this is random, not systematic.

An equally dramatic situation is the battle of tidal-stretching versus gravitational accumulation, which can occur in the centers of bridges. This is illustrated in the Arp 305 system, where a TDG seems to be forming in the bridge center (see 4 and Hancock, et al. 2009). Similarly, there are the objects my collaborators have named Hinge Clumps (see Hancock, et al. 2009, and Smith 2010b). These are luminous star forming regions found in disks at the base of tidal tails. Examples are shown in Fig. 5.

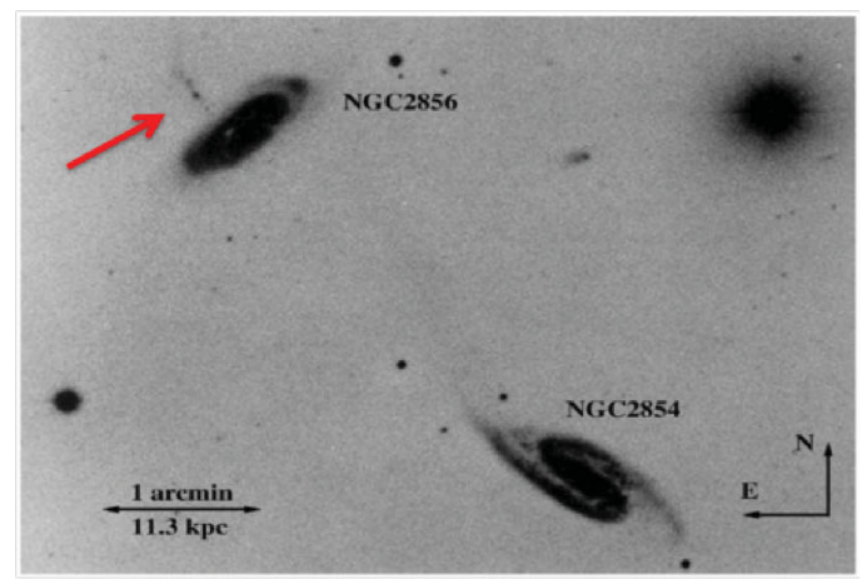

Figure 3. Optical image of the NGC 2854/2856 system (from Smith, et al. 2008). The northern galaxy has a rather faint "beads-on-a-string" plume, which models indicate may be a pileup zone of material transferred from the southern galaxy. 
In sum, ISF in galaxy collisions comes in a much wider range of variations on themes of compression than in isolated disks. The dominant subthemes are the same: shocks, gravitational collapse/fragmentation and accretion. Another thing that is the same in all the various environments is that star formation occurs in clusters, and often in clusters of clusters. There seems to be wide consensus that the cluster birth function is universal across interacting and isolated galaxies. Currently, there is some debate about cluster disruption processes and timescales. Observations of interacting galaxies have provided input into this debate, but attention has been strongly focused on a few nearby systems (especially M51 and the Antennae), which do not sample the full range of star-forming environmental conditions in interactions. Studies of other nearby systems can provide more input. For example, Peterson, et al. (2009) find that some very young groups of star clusters in the Arp 284 system are embedded in a halo of intermediate age stars. This can tell us about the disruption SF/disruption history of such regions.

\section{Concluding Miscellany and Prospects}

In order to begin to understand the mechanisms of induced SF in the many environments of interacting galaxies, dynamical models of prototypical systems, or if the data are of high enough quality, models of individual systems are needed. In the latter case, such models have traditionally been handcrafted, i.e., fit by trial and error. With aid of some general rules of thumb the art has become quite well developed. Even so, the future promises broader developments.

In the last decade, several groups have attempted automatic modeling in the sense that the simulation code is able to explore models with different collision parameters, seek

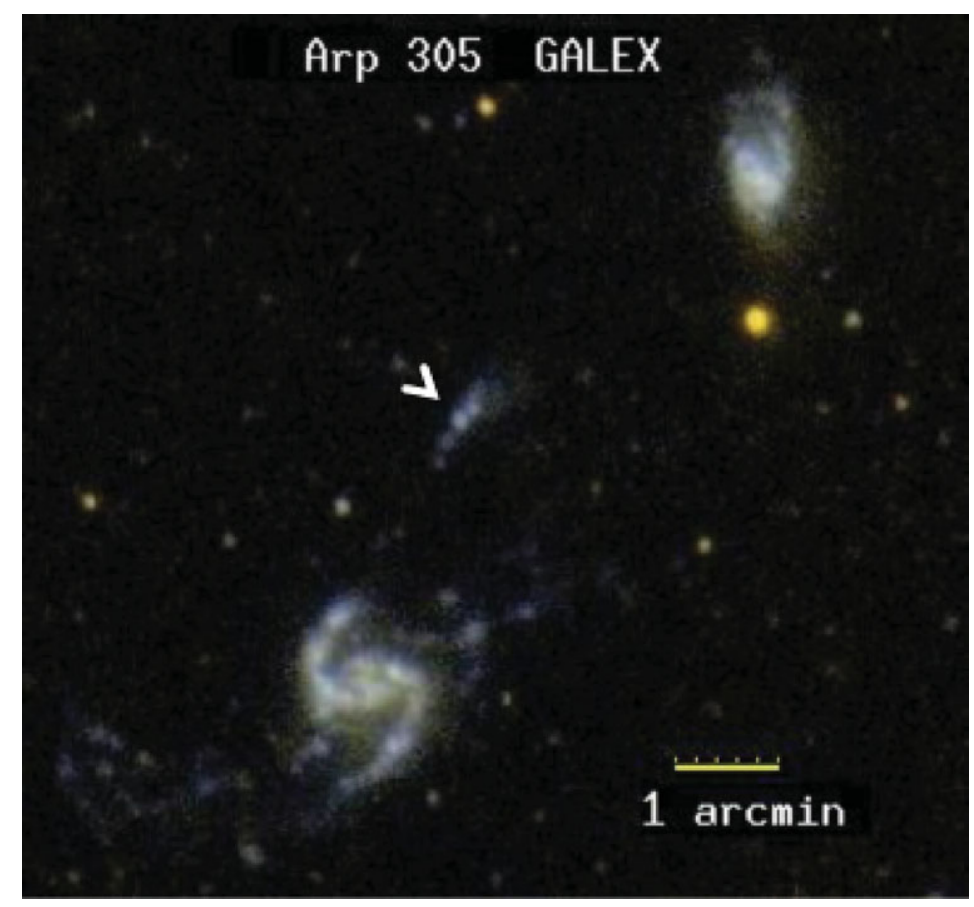

Figure 4. A candidate TDG forming in the bridge between the two galaxies of Arp 305, as revealed in GALEX observations (from Hancock, et al. 2009). In published observations HI observations gas is seen to connect the two galaxies, confirming the existence of the bridge. 
best fit models, and then use these as the basis for another round of modeling and fitting (see Smith, et al. 2010c and references therein, also Gomez, et al. 2004). Most of these works use genetic algorithms. Fitting detailed structures of ISF has not been attempted, only morphological and velocity structures. The difficulty of this procedure grows rapidly with the number of the observational constraints and their resolution. Interestingly, the goodness-of-fit measure does not increase smoothly, but rather in jumps. At present, alternatives include "expert-learning" systems like the Barnes/Hibbard Identikit (Barnes \& Hibbard 2009) or Chilingarian et al.'s GalMer database (Chilingarian, et al. 2010).

I also believe that analytic work could be further developed in this field, at least to aid in understanding complex numerical simulations. For example, analytic descriptions of waves have proven feasible and useful in studies of colliding ring galaxies. The study of caustic waveforms can applied more generally (see e.g., Struck-Marcell 1990, Gerber \& Lamb 1994). Simple, restricted 3-body modeling is very useful for impulsive encounters, and is nearly semi-analytic in such cases.

Models for multiple interactions in specific groups have not been attempted often. The complexity is great and the collision parameter space is dauntingly large. A significant fraction of cosmic star formation occurs in such environments, and more so at high redshift. This provides motivations for studying local examples in detail. Cosmological structure simulations provide much information about SF in multiple mergers, but not all that we would like. Are there general rules-of-thumb governing the dynamics of such systems?

J.-S. Hwang and I (in collaboration with F. Renaud and P. N. Appleton) are computing models of the evolution of Stephan's Quintet involving multiple collisions to better understand these processes. One simplification: we find that most of the major features can be modeled as the result of a series of 4 close 2-body encounters. A complication: initial conditions of successive 2-body encounters are not in any kind of steady state! These conclusions are probably general.

With work proceeding on so many fronts, and the computational tools to deal with increased complexity, there is good reason to believe that our understanding of interaction induced SF will advance considerably in the next decade. Prominent interacting systems

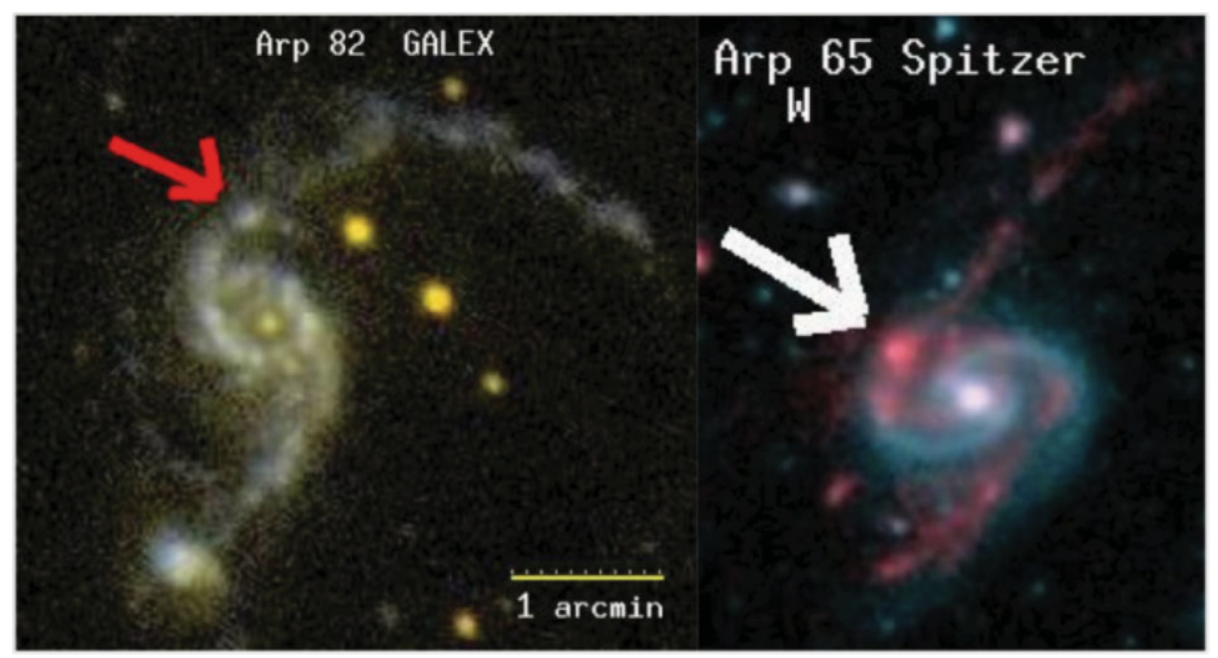

Figure 5. Two examples of "hinge clumps" in Arp 65 and Arp 82 (from Smith 2010b). Hinge clumps are prominent knots of star formation at the base of a tidal tail, but within the galaxy disk. 
are not located nearby, so their study is at a more exploratory stage than Galactic or Local Group studies described at this conference. However, the foundation is being laid for more exciting work in the future.

\section{References}

Appleton, P. N., Charmandaris, V., Gao, Y., Jarrett, T., \& Bransford, M. A. 2003, ApJ, 586, 112

Barnes, J. E. \& Hernquist, L. 1996, ApJ, 471, 115

Barnes, J. E. \& Hibbard, J. E. 2009, AJ, 137, 3071

Bournaud, F. \& Duc, P.-A. 2006, A\&A, 456, 481

Chien, L.-H. \& Barnes, J. E. 2010, MNRAS, 407, 43

Chilingarian, I., Di Matteo, P., Combes, F., Melchoir, A.-L., \& Semelin, B. 2010, A $\mho A, 518,61$

Duc, P.-A., Bournaud, F., \& Masset, F. 2004, A\&A, 427, 803

Efremov, Y. N. 2010, MNRAS, 405, 1531

Elmegreen, B. G. 1989, ApJ (Letters), 342, L67

Elmegreen, D. M., Elmegreen, B. G., Kaufman, M., Sheth, K., Struck, C., Thomasson, M., \& Brinks, E. 2006 ApJ 642, 158

Gao, Y., Zhu, M. \& Seaquist, E. R. 2003, AJ, 126, 2171

Gerber, R. A. \& Lamb, S. A. 1994, ApJ, 431, 604

Gomez, J. C., Athanassoula, L., Fuentes, O., \& Bosma, A. 2004, in F. Ochsenbein, M. G. Allen, \& D. Egret (eds.), Astronomical Data Analysis Software and Systems (ADASS) XIII: A.S.P. Conf. Series, 314 (San Francisco: ASP), p. 629

Hancock, M., Smith, B. J., Struck, C., Giroux, M. L., \& Hurlock, S. 2009, AJ, 137, 4643

Jogee, S., et al. 2009, ApJ, 697, 1971

Kaufman, M., Grupe, D., Elmegreen, D. M., Elmegreen, B. G., Struck, C., \& Brinks, E. 2010, $A J$, in preparation

Kim, J., Wise, J. H., \& Abel, T. 2009, ApJ, 694, L123

Lisenfeld, U., Bournaud, F., Brinks, E., \& Duc, P.-A. 2009, astro-ph, 0903-0999

Mihos, J. C. \& Hernquist, L. 1996, ApJ, 464, 641

Peterson, B. W., Struck, C., Smith, B. J., \& Hancock, M. 2009, MNRAS, 400, 1208

Saitoh, T. R., Daisaka, H., Kokubo, E., Makino, J., Okamoto, T., Tomisaka, K., Wada, K., \& Yoshida, N. 2009, PASJ, 61, 481

Shi, Y., Rieke, G., Lotz, J., \& Perez-Gonzalez, P. G. 2009, ApJ, 697, 1764

Smith, B. J., et al. 2008, AJ, 135, 2406

Smith, B. J., Bastian, N., Higdon, S. J. U., \& Higdon, J. L. (eds.) 2010a, Galaxy Wars: Stellar Populations and Star Formation in Interacting Galaxies: A.S.P. Conf. Series, 423 (San Francisco: ASP)

Smith, B. J., et al. 2010c, in Smith, B. J., Bastian, N., Higdon, S. J. U., \& Higdon, J. L. (eds.), Galaxy Wars: Stellar Populations and Star Formation in Interacting Galaxies: A.S.P. Conf. Series, 423 (San Francisco: ASP), p. 227

Smith, B. J., Giroux, M. L., Struck, C., Hancock, M., \& Hurlock, S. 2010b, AJ, 139, 1212

Struck, C. 2005, MNRAS, 364, 69

Struck, C. 2006, in: J. W. Mason (ed.), Astrophysics Update 2 (Heidelberg: Springer Praxis), p. 115

Struck-Marcell, C. 1990, AJ, 99, 71

Sulentic, J. W., Keel, W. C., \& Telesco, C. M. (eds.) 1990, Paired and Interacting Galaxies: I.A.U. Colloq. 124 (Washington, D. C.: NASA)

Teyssier, R., Chapon, D., \& Bournaud, F. 2010, ApJ, 720, L149 